\title{
Intracranial Mature Teratoma in an Adult Patient: A Case Report
}

\author{
Dominik Romić ${ }^{1}$ Marina Raguž ${ }^{1}$ Petar Marčinković ${ }^{1}$ Patricija Sesar ${ }^{2}$ Martina Špero ${ }^{3}$ \\ Zrinka Čolak Romić ${ }^{4}$ Domagoj Dlaka ${ }^{1}$ Darko Chudy ${ }^{1}$ \\ ${ }^{1}$ Department of Neurosurgery, University Hospital Dubrava, Zagreb, \\ Croatia \\ 2 Department of Pathology and Cytology, University Hospital \\ Dubrava, Zagreb, Croatia \\ ${ }^{3}$ Department of Radiology, University Hospital Dubrava, Zagreb, \\ Croatia \\ ${ }^{4}$ Department of Neurology, University Hospital Dubrava, Zagreb, \\ Croatia \\ J Neurol Surg Rep 2019;80:e14-e17. \\ Address for correspondence Marina Raguž, MD, PhD, Department of \\ Neurosurgery, University Hospital Dubrava, Avenija Gojka Suska 6 \\ Zagreb HR-10000, Croatia (e-mail: marinaraguz@gmail.com).
}

\begin{abstract}
Keywords

- mature teratoma

- adult

- intracranial teratoma

Introduction: Primary intracranial teratoma is a subtype of germ cell tumors, classified into three subtypes. They occur very rarely, with only several reported individual cases in adults.

Case Description We present a patient with an intermittent headache in the right frontal region. Magnetic resonance imaging (MRI) revealed a right sided high frontal parasagittal mass that compressed the falx, the right lateral ventricle, as well as the brain parenchyma. Patient underwent surgical treatment. Histopathological analysis described mature teratoma. Four months after the surgical treatment there were no signs of residual intracranial mass or relapse.

Discussion Primary intracranial teratoma in adults has a nonspecific clinical presentation. MRI reveals a solitary irregular mass with multilocularity and mixed signals derived from different tissues. The patients age, biochemical markers, and patohistological analysis are necessary to confirm the diagnosis.

Conclusion Teratoma treatment strategy still remains controversial. It includes radical resection whenever possible. Since the residual portion of mature teratoma may contain part of immature or malignant tissue, tumor recurrence after surgical removal is possible. Also, new tumor mass could occur at other sites intracranial after the initial one was removed. Thus, although patients usually recover, they should be followed-up for a long period of time.
\end{abstract}

\section{Introduction}

Central nervous system germ cell tumors (GCT) are very rare, comprising approximately 0.3 to $0.6 \%$ of all primary intracranial tumors, ${ }^{1,2}$ and they can be divided in following classes: germinomas, nongerminomatous (teratoma, embryonic carcinoma, endodermal sinus tumor, choriocar-

received

September 23, 2018

accepted

January 18, 2019
DOI https://doi.org/

$10.1055 / \mathrm{s}-0039-1685213$. ISSN 2193-6358. cinoma), and mixed GCT. ${ }^{2-4}$ Teratomas are a subtype of nongerminomatous GCT, which originate from pluripotent cells, which in turn are derived from all three germ cell layers present in normal organogenesis. The majority of reported teratomas contain tissue from all three germ cell layers (endoderm, mesoderm, and ectoderm). However, they occasionally develop from a single germ cell layer if they show
License terms

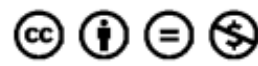

(c) 2019 Georg Thieme Verlag KG Stuttgart · New York 


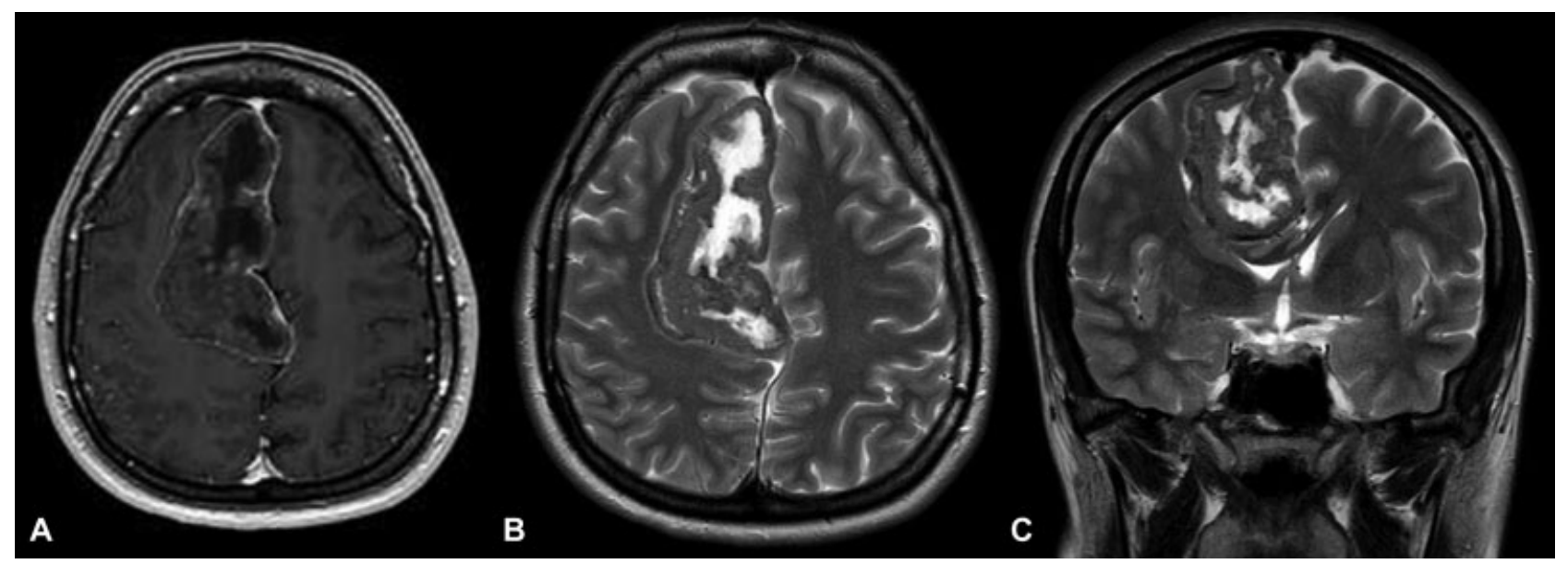

Fig. 1 Initial brain MRI showing right sided extra axial high parietal parasagittal mass in (A) transverse plain, T1-weighted image; (B) transverse plain, T2-weighted image; and (C) coronal plain, T1-weighted image. Note the marginal heterogeneous enhancement of the tumor after the administration of intravenous contrast (A). MRI, magnetic resonance imaging.

histological divergent differentiation. ${ }^{2}$ Teratomas are hypothesized to arise by a misplacement of a pluripotent germ cell. Primary intracranial teratomas occur very rarely, with only several individual reported cases in adults. Several articles report that intracranial teratomas usually present in patients between 1 and 46 years of age (mean, 14.8 years) and have a male predominance (male to female ratio, 28:3). ${ }^{5}$

According to histological findings, teratomas are classified into three subtypes: mature teratomas, immature teratomas, and teratomas with a malignant transformation. ${ }^{1-3,6}$ Mature teratomas are mainly composed of well-differentiated tissue with low mitotic activity, while immature teratoma consist of components resembling fetal tissue like hypercellular embryonic mesenchyme or primitive neuroectodermal elements that can be mixed with mature tissue elements. Teratomas with malignant transformation are extremely rare and are characterized with additional malignant somatic tissue. ${ }^{1,7}$ Teratomas, as well as GCT, are usually located in the midline structures of the brain since the midline is a location with a great potential for misplacement of embryonic tissues. Teratomas can thus be usually found in areas, such as around the third ventricle, especially the pineal region, and the suprasellar or parasellar region. Less common sites include the ventricular system, cerebellar vermis, basal ganglia, cerebellopontine angle, and the cavernous sinus. ${ }^{1,6}$

\section{Case Description}

A 37-year-old female patient presented with a 4-month history of intermittent headache in the right frontal region. There were no other symptoms nor were there any neurological deficits at the time of admission. Initial computerized tomography (CT) and magnetic resonance imaging (MRI) revealed a right sided extra-axial frontal parasagittal mass measuring $8.5 \times 4.2 \times 6.1 \mathrm{~cm}$ with several calcified areas and a central necrotic part. The mass compressed the surrounding brain parenchyma, especially primary motor cortex, partially the right lateral ventricle, and also the superior sagittal sinus, without infiltrating the aforementioned struc- tures. Also, there was invasion and destruction of cerebral falx. After the administration of intravenous contrast, the mass showed marginal heterogeneous enhancement (-Fig. 1). According to the MRI scan, differential diagnoses included meningioma, fibrous tumor of the dura mater or other primary process of the dura.

Patient underwent a right sided parasagittal frontoparietal craniotomy. A mostly calcified mass with several central soft areas that was invading and destroying a large part of the cerebral falx (with no invasion to the superior sagittal sinus) was completely removed. Partial duroplasty was performed. Surgery went without any complications. A control CT scan, performed on the first postoperative day, revealed a satisfactory finding, without any signs of ischemia, hemorrhage, or residual tumor. Patient recovered uneventfully. Tissue samples acquired during the surgical procedure underwent patohistological analysis. The tumor consisted of mature cartilage tissue, several fragments of bone tissue, and mature adipose tissue (-Fig. 2). According to the WHO (World Health Organization) classification, it corresponded to a mature teratoma. An oncologist was consulted. There was no indication for further oncological treatment and the patient afterwards underwent regular radiological follow-up.

Four months afterwards, an MRI as well as a further neurosurgical control was performed. Patient was without any neurological deficits or headaches. MRI revealed a satisfying postoperative result without recurrence of intracranial masses (-Fig. 3).

\section{Discussion}

Teratomas are usually seen as a solitary irregular mass with or without multilocularity and mixed signals derived from different tissues. After intravenous contrast administration teratomas usually show heterogeneous enhancement on the MRI, while its internal architecture is best displayed on T1- and T2-weighted images. Perilesional cerebral edema isn't usually present, due to the presence of the tumor 

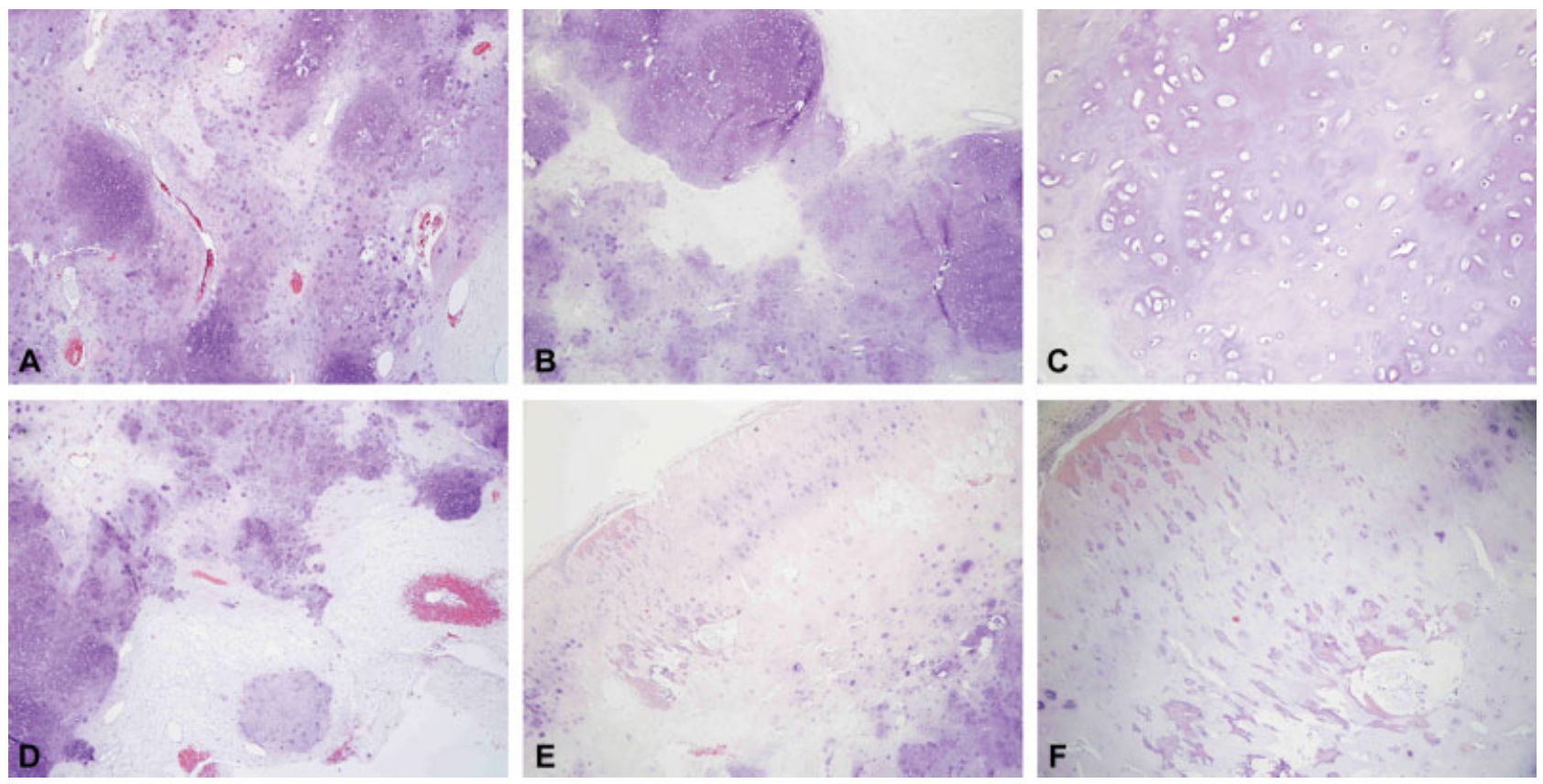

Fig. 2 Microphotography of a patohistological section of a removed teratoma stained with hemalaun eosin: (A and B) cartilage, magnification of $\times 40$; (C) cartilage, magnification of $\times 200$; (D) cartilage and adipose tissue, magnification of $\times 40$; (E) bone and cartilage, magnification of $\times 40$; and $(\mathrm{F})$ bone and cartilage, magnification of $\times 100$.

capsule as well as the undamaged blood brain barrier. MRI signal intensity reflects the various types of tissue within teratomas, such as fibrosis, adipose tissue, calcification, cystic elements, hair follicles, cellular debris, and keratinocytes. ${ }^{1,2}$

Clinically, the incidence of teratomas decreases with age. The patient's clinical presentation is nonspecific, depending on the tumor size and location, with signs of increased intracranial pressure as the most common one., ${ }^{1,6}$

Before the diagnosis of a teratoma is established, it is important to exclude other intracranial neoplasms. The patient's age and biochemical markers, such as serum alphafetoprotein (AFP) and $\beta$-human chorionic gonadotropin (bHCG), can be useful in differentiating teratomas from other intracranial neoplasms. If elevated, serum AFP levels are strongly indicative of a malignant teratoma. Spotted calcification and adipose components revealed using CT or MRI scans are characteristic for intracranial teratoma diagnosis. ${ }^{1,6,7}$ Definitive diagnosis is confirmed using a patohistological finding. As mentioned earlier, teratomas are divided into three further subtypes depending on the maturity of their tissue. In addition to the mature and immature subtypes, teratomas can also manifest a great number of mitoses in an immature tissue sample, indicating a teratoma with a malignant transformation. ${ }^{6}$ It is of great importance to determine the exact patohistological diagnosis using extensive sampling, as immature tissue can constitute even a minor part of the tumor and therefore direct to another treatment option., ${ }^{8,9}$

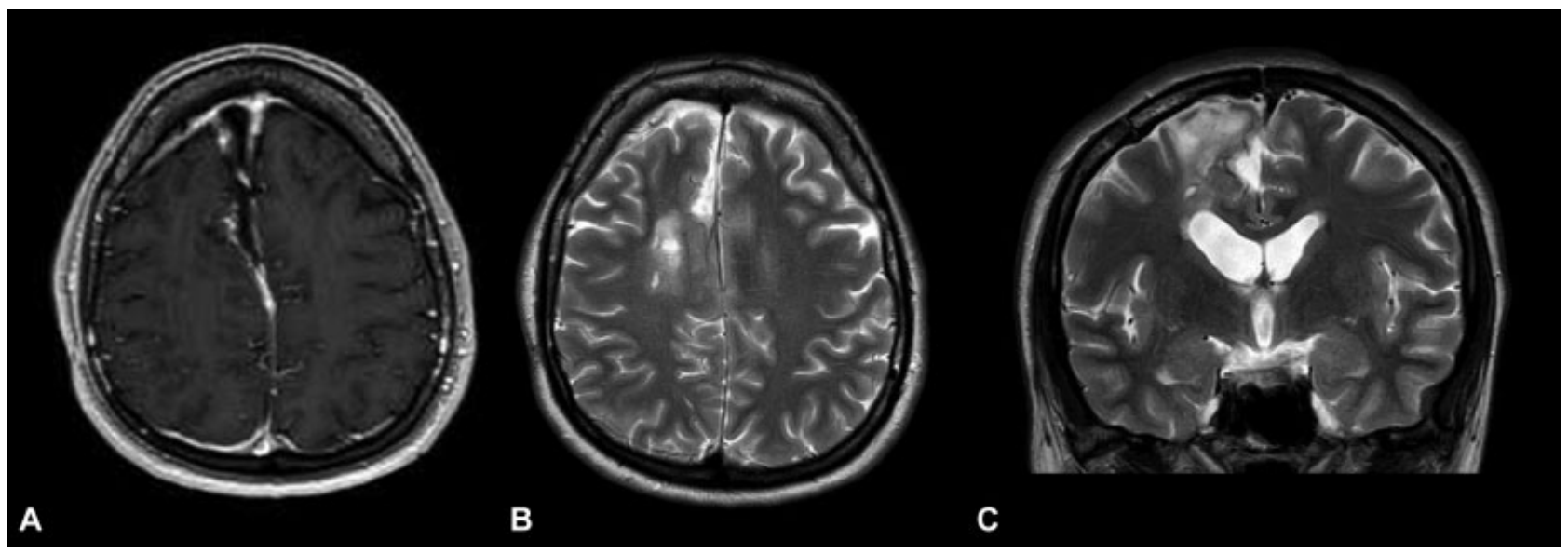

Fig. 3 Brain MRI scans, four months postoperatively, (A) transverse plain, T1-weighted image; (B) transverse plain, T2-weighted image; (C) coronal plain, T1-weighted image. Note how there is no recurrence or additional intracranial mass in the images. MRI, magnetic resonance imaging. 
There are two main theories that could explain the pathogenesis of GCT in the central nervous system. While the germ cell theory suggests that teratomas arise from primordial germ cells which migrated during embryogenesis and underwent malignant transformation, the embryonic theory suggests that teratomas arise from a mismigrational pluripotent germ cell. ${ }^{6,7}$ Since the brain midline structures are mostly affected with teratomas, and since the cerebral falx, the cavernous sinus walls, as well as the septum pellucidum are histological the same as the dura mater, it has been suggested that the abnormal migration of primordial germ cells into the dura mater might be responsible for the occurrence of intracranial mature teratomas. ${ }^{7}$ It has also been hypothesized that this kind of migration is under the control of complex molecular events. ${ }^{6}$

Several reported cases showed that new teratomas could occur at other intracranial sites after an initial one was removed. These cases suggest that the pathogenesis of teratomas might be associated with genetic alterations. Experimental analyses demonstrated that teratomas harbored a genetic loss or mutation, such as the loss of heterozygosity in the E-cadherin gene and the mutation in the exon- 3 of the $\beta$ catenin gene, which are components of adherent junctions. Morphological changes that occur as a consequence of genetic changes could be an important factor in tumorigenesis. ${ }^{7,10}$

So far, teratoma treatment strategies still remain controversial. Treatment includes radical resection whenever possible, with surgical approach depending on the location of the teratoma. Excision is considered complete if more than $90 \%$ of the tumor has been removed. ${ }^{6}$ As mature teratomas are considered radioresistent, total tumor removal is the treatment of choice. ${ }^{4}$ The clinical outcome in patients with primary intracranial teratomas is dependent on the pathology. Since any residual portion of a diagnosed mature teratoma may also contain a small part of immature or malignant tissue, recurrence after its partial removal has indeed previously been reported. Previous studies report a 5year survival rate for patients with an immature or mature teratoma with a malignant element in between 44 and $68 \%{ }^{7}$ Although mature teratoma has a low recurrence rate after surgical resection, various articles report occurrence of other GCTs on different intracranial sites. ${ }^{11,12}$ While patients usually recover uneventfully, they should be followed-up for a longer period, since malignant teratomas or new teratomas may arise intra- or extracranially.

\section{Conclusion}

We reported a case of a patient with a mature teratoma that destroyed a large part of the cerebral falx which in fact can also be its starting point. This observation can go in favor of abnormal migration of primordial germ cells into the dura or better said in favor of the embryonic theory. Radical resection, whenever possible, is the treatment option for teratomas. As new or recurrent teratomas (or other GCT) might appear, a long period follow-up is essential in patients that undergo surgical procedure for intracranial teratoma.

\section{Compliance with Ethical Standards}

The patient has given an informed consent for participation in this paper.

\section{Conflict of Interest}

On behalf of all authors, the corresponding author states that there is no conflicts of interest.

\section{References}

1 Georgiu C, Opincariu I, Cebotaru CL, et al. Intracranial immature teratoma with a primitive neuroectodermal malignant transformation - case report and review of the literature. Rom J Morphol Embryol 2016;57(04):1389-1395

2 Liu Z, Lv X, Wang W, et al. Imaging characteristics of primary intracranial teratoma. Acta Radiol 2014;55(07):874-881

3 Kong Z, Wang Y, Dai C, Yao Y, Ma W, Wang Y. Central nervous system germ cell tumors: a review of the literature. J Child Neurol 2018;33(09):610-620

$4 \mathrm{Kim}$ JM, Cheong JH, Yi HJ, Bak KH, Kim CH, Oh SJ. Metachronous germinoma after total removal of mature teratoma in the third ventricle: a case report. J Korean Med Sci 2002;17(02):287-291

5 Lee YH, Park EK, Park YS, Shim KW, Choi JU, Kim DS. Treatment and outcomes of primary intracranial teratoma. Childs Nerv Syst 2009;25(12):1581-1587

6 Agrawal M, Uppin MS, Patibandla MR, et al. Teratomas in central nervous system: a clinico-morphological study with review of literature. Neurol India 2010;58(06):841-846

7 Zhao J, Wang H, Yu J, Zhong Y, Ge P. Cerebral falx mature teratoma with rare imaging in an adult. Int J Med Sci 2012;9(04):269-273

8 Dimov I, Tasić D, Stojanović I, et al. Mature intracranial teratoma. Acta Fac Med Naiss 2013;30(02):97-102

9 Beschorner R, Schittenhelm J, Bueltmann E, Ritz R, Meyermann R, Mittelbronn M. Mature cerebellar teratoma in adulthood. Neuropathology 2009;29(02):176-180

10 Pećina-Slaus N, Niku Eva-Martić T, Beros V, Tomas D. Genetic alterations of E-cadherin and beta-catenin in germinoma and teratoma: report of two central nervous system cases. Pathol Oncol Res 2007;13(04):370-374

11 Sugimoto K, Nakahara I, Nishikawa M. Bilateral metachronous germinoma of the basal ganglia occurring long after total removal of a mature pineal teratoma: case report. Neurosurgery 2002;50 (03):613-616, discussion 616-617

12 Janzarik WG, Müller K, Lübbert M, et al. Occurrence of a germinoma 22 years after resection of a mature cerebral teratoma. J Neurooncol 2008;88(02):217-219 